\title{
Factor Affecting Coffee (Coffee Arabica L) Quality and Grading Methods
}

\author{
Sewmehon Alemnew ${ }^{1} \quad$ Geletaw Kebede ${ }^{2}$ \\ College of Agriculture, Department of horticulture, Wolaita Sodo University, \\ P.O.BOX : 138 , Wolaita Sodo, Ethiopia
}

\begin{abstract}
The coffee production in Ethiopia is the critical to Ethiopian economy with about quarter of total population depending directly or indirectly on their live hood. But, coffee production in Ethiopia is mainly concentrated in the south, southwest and east of the country. The annual production ranges from 200,000 to 250,000 metric tons depending on weather and soil condition. Coffee is produced by four methods in Ethiopia: forest coffee, semiforest coffee, garden coffee and plantation coffee which they account $10 \%, 35 \%, 50 \%$ and $5 \%$, respectively. Despite favorable climatic conditions variety of local coffee types for quality improvement and long history of production in Ethiopia, coffee quality is declining from time to time due to several improper pre and post-harvest management practice. To improve quality of coffee appropriate agronomic and envirmental factors were paramount importance. Therefore growing of coffee in suitable climactic condition and used adopted cult varies were indispensable to have quality coffee so as to increase income of the growers and the country as a whole.
\end{abstract}

DOI: $10.7176 / \mathrm{JNSR} / 10-4-02$

Publication date: February $29^{\text {th }} 2020$

\section{INTRODUCTION}

Coffee (Coffee arabica) is a nonalcoholic stimulant beverage crop that belongs to the family of Rubiaceae and genus Coffea. Among more than 100 species of coffee, Coffea arabica, Coffee canephora and Coffea libercia of are the most economically important species worldwide (Mabbarely, 1997).

Coffee Arabica is believed to originate in humid high rain forests of south and south western Ethiopia. In general coffee is the world most important internationally traded commodity in terms of monetary value after petroleum and primary exports of many developing countries. Coffee ranked as the fifth most important trade commodity after wheat, cotton, maize, and rice (FAO, 2008). Ethiopia is a major source of genetic resources for origin and diversity of coffee Arabica. It is cultivated in most parts of tropics, accounting for 80 percent of the world coffee market. The crop plays an important role in income and employment in developing countries like Africa, Asia and Latin America (Behilu et al., 2008).

Coffee is the major sources of foreign currency income for Ethiopia and contributes more than 35 percent of total export earnings. Thus, it is a comers stone in the export economy of country and it directly or indirectly 25 percent of the live hood of total population (Fayera, 2006). Ethiopia is well known not only for being the home of Arabica coffee, but also for its very fine quality coffee acclaimed for its aroma and flavor characteristics. The coffee that are disStinguished for are such unique characteristics include Sidamo, Yirgachafe, Harar, Gimbi and Limmu coffee types (Anwar, 2010).

However, Workafes and Kebede (2000) stated that coffee produced in some parts of Ethiopia, especially from Harar and yirgachafe are always sold at a premium prices both at domestic and international coffee markets because of its distinctive fine quality and appropriate processing approach. Coffee grows under diverse environmental condition ranging from 550 to $2600 \mathrm{~m}$ above sea level, with annual rain fall from 1000 to $2000 \mathrm{~mm}$ and the temperature (minimum and maximum range from 8-150C and 24-30 0C respectively (Willson, 1999). Even though there is a suitable climatic condition under Ethiopian condition, generally coffee quality exported to abroad or locally consumption is not satisfactory as it has to be. In view of the present situation, making effort to overcome challenges and threat slowly through expansion of production does not seem visible for the country. Thus, it has been repeatedly mentioned at various forum that providing good quality coffee is the only way out and viable option to get in to world market and to remain help producers to hedge their marketing risks (Behailu et al., 2008).

Despite favorable climatic conditions, high genetic diversity of coffee Arabica for quality improvement and long history of production in Ethiopia, coffee quality is declining from time to time due to several improper pre and post-harvest management practice. This still practiced by the majority of coffee producers/farmers, from which larger proportion of the product is obtained; these quality problems are mainly associated with poor agronomic practices, like lack of stumping, pruning and weeding (Tena, 2008).

Others factors like climate, genetic makeup of plants and institutional also affect coffee production, productivity and finally coffee quality. However, there is wide gap in review and documentation of factors affecting coffee quality in Ethiopia like climate, pre and post-harvest, soil condition of coffee growing area and genetic makeup of coffee plants. Therefore, the objective of these seminar is to review the factors affecting coffee 
Arabica quality and grading methods.

\subsection{Statement of the problem}

Coffee culture is now in a growing trend where there are more coffee drinkers enjoying having a cup of coffee in the coffee shop. The consumers nowadays contributed more by the younger generations as they perceive coffee not just a beverage, but a self-ego. This is where socialization plays an important role for them. They tend to have gathering or study discussion with their friends in those coffee shops that provide a comfortable environment. Choosing a coffee shop for these coffee drinkers nowadays does not only look into the quality of coffee, but also takes into consideration of other factors such as service quality provided by the coffee shop.

Due to the growing demand, International Coffee Chains in Malaysia are on a rise. However, there is insufficient study for Malaysian context particularly in the coffee industry that is related to service quality, servicescape, customer satisfaction and behavioral intentions. As most of the research done in Malaysia are more to fast food industry, but service quality and service scape are still very important to coffee industry in the service field. There is also an inconsistency in service quality and service scape provided by these International Coffee Chains. These coffee chains are not delivering each of the dimensions in the service quality and service scrape up to the customers' expectation. Thus, it leads to customer dissatisfaction and eventually affects the behavioral intentions. Therefore, there is a need to study on the service quality and service scape on customer satisfaction towards behavioral intentions in the International Coffee Chains.

Service quality plays an important role especially in food and beverage industry. Good service quality will give a good impression to customers and lead to repeat visit by customers. However, some coffee shop ignores to perform a well service quality to customers. Few elements will be discussed in this study including tangibles, reliability, responsiveness, assurance and empathy.

\subsection{Objectives}

To an over review factors that affects Coffee quality and grading methods

\section{LITERATURE REVIEW}

\subsection{Coffee Arabica Production}

More than 125 countries are involved in coffee production, trading and communication of the product. Out of this, more than 50 developing countries are earning 25 percent of their foreign exchange from coffee production and export (Fayera, 2006). Coffee production in under Ethiopia condition is the critical to Ethiopian economy, as about quarter of total population depending directly or indirectly for their live hood. Now days, Ethiopia is the world third and Africa's top producers of coffee Arabica (ICO, 2011).

However, half of the coffee is consumed by Ethiopians locally and country leads continent in domestic consumption. The total areas used for coffee cultivation is estimated to be about 400,000 hectars, but the size is unknown due to the fragmented nature and intercrops of coffee farms. The majority of coffee production in Ethiopia 90 percent comes from the small holders, while the rest is produced and exported by large scale producers or start farms and inventors (Taye and Tesfaye, 2002).

Coffee production in Ethiopia is mainly concentrated in the south, southwest and east of the country. The annual production ranges from 200,000 to 250,000 metric tons depending on weather and soil condition (Tena, 2008). The potential for coffee Arabica production in Ethiopia is very high due to suitable altitude, ample rain fall, optimum temperature planting materials and fertile soil. Furthermore the country is of particular interest to the world, because it is where Coffee arabica originated and thus has the best inherent quality of coffee production potential.

Coffee is produced in Ethiopia in different areas like Jimma, Iluabor, Kaffa, Hararghe, Wellega, Sidamo, Yirgachafe and others. But, coffee grows at various altitudes that range from 550-2600m above sea level. However, Abiyot (2008) stated that coffee Arabica grows in the eastern, southern and western parts of country with altitude ranging between 1300 to $1800 \mathrm{~m}$ above sea level and the annual rain fall in the coffee growing regions of the country varies between $1500 \mathrm{~mm}$ to $2500 \mathrm{~mm}$. The ideal temperature for coffee Arabica production is $15^{\circ} \mathrm{C}-30^{\circ} \mathrm{C}$ and this temperature prevails in most coffee growing areas of Ethiopia and coffee production require fertile soil ,friable loam soil with 1.5 of depth.

\subsubsection{Coffee production systems}

Under Ethiopia condition, coffee produced in four different systems as: forest coffee, semi-forest coffee, garden coffee and plantation coffee. 95 percent of the coffee production from these systems can be considered as organic, although not yet officially certified (Workafes and kassu, 2000).

Forest coffee: It is found in south and south western of Ethiopia like bale, west Wollega, Bench-Maji, Keficho, Shekicho, Metu and Jimma. These are the center of coffee Arabica origion.Forest coffee is self-sown and grown under the shade of natural forest trees. Forest coffee offers a wide diversity for selection and breeding so as to have plant stock selected for disease resistance, high yields and top quality in terms of aroma as well as flavor. It 
accounts about 10 percent of total coffee production (Workafes and kassu, 2000).

Semi forest coffee: production system found in the southern and southwestern parts of the country. It accounts about 35 percent of total coffee production.

Garden coffee: is found in the vicinity of farmers' residences. It is mainly found southern and eastern parts of our country like Haraghe, Gedeo, southern and northern Omo, Sidamo, Wollega and Gurage zones. This production system also accounts 50 percent of total coffee production, and now days increases in the south west Ethiopia like Kaficho, Shekicho and Benji-Maji.

Plantation coffee system is coffee grown and owned by the state farms and large scale sale privatization farms. For this production system, recommended agronomic practice, like improve seedling, spacing, proper planting, mulching, manuring, shade regulation and pruning have to be practiced. It accounts 5 percent of total production (Tena, 2008).

\subsubsection{Coffee quality}

The quality of coffee is a combination both genetic makeup of the plant and environmental condition including human involvement in production and processing, finally affects flavor and aroma of the cup quality. These come from the chemical constituents of the coffee beans. The chemical reaction takes place during processing, particularly in the fermentation stage of wet processing, which maintain the desired chemicals. The quality of cherries influence the quality of product; under ripe, over ripe, diseased and damage cherries reduces quality. The final assessment of quality is made subjectively by skilled taster who considers raw analysis and organoleptic quality. The quality assessed has a marked influenced on the prices at which coffee can be sold (Willson, 1999).In general, the international organization for standardization (ISO) describes quality as the ability of set of inherent characteristics of product, system or process to fulfill requirements of customers and other interested parties.

\section{1 .3 Major factors affecting coffee arabica quality}

Coffee quality is a complex characteristic which depends on series of factors such as the species, genetic factors, environmental condition, storage conditions, industrial processing and preparation of the beverages and taste of the consumers. Coffee quality is critical important to the coffee industry. In general coffee quality determined by different factors, those includes: genotype, climatic condition, soil characteristics of the area, agronomic practices, harvesting time, post-harvest processing techniques, grading, packing, storage condition and transport all contributes either exaltation or deterioration of coffee quality (Girma et al., 2007).

\subsubsection{Climatic and soil factors}

Alemayehu et al (2008) reported that environment factors like altitude, daily temperature fluctuation, amounts and distribution of rain fall, physical and chemical characteristics of soil have a strong influence on coffee quality. Climate, altitude and shade play important role though temperature and rain fall affect coffee during ripening and flowering bean expansion (Fayera, 2006). The slowdown ripening process of coffee berries at higher elevation (low air temperature) or under shading allows more time for complete bean filling. The slow maturation process should therefore play critical role in determining high cup quality, possible by guarantying the full manifestation of all biochemical steps required for the development of beverage quality (Bezawit, 2011).The role of soil types has been study is grown on rich volcanic solids.

The perceived acidity of coffee brews has always been recognized as an important attribute of coffee quality. However, is an extreme of acidity can be considered as defects (Wiley and Sons, 2001). Acidity has been correlated with coffee grown at very high altitudes and in mineral rich volcanic soil. On the other hand Fayera (2006) reported that if other factor kept constant, better quality can be found at high altitudes, while low land coffee were found to be somewhat bland with considerable body. Moreover coffee from high altitudes areas is more acidic with better aroma and flavor.

The total rain fall, relative humidity with maximum and minimum effects on water vapor content of air and storage durations greatly influences storability and quality of stored parchment coffee and the period of prolonged may also result in lower quality beans (Girma et al ,2007)

\subsubsection{Pre-harvest and harvest management factors}

Coffee grown with heavy application of nitrogen fertilizers had poorer, lighter and thinner quality than unfertilized fields. An excess of nitrogen increases caffeine content, resulting in more bitter taste of the brew. The caffeine and chlorogenic acid contents of the beans are not much affected by the levels of phosphorus, calcium, potassium and magnesium in the soil (Wiley and Sons, 2001).

Adugna (2007) reported that shades tree ideally not improve cup quality on the contrary indicated that shade improves the appearances of green and roasted coffee beans as well as acidity and body of the brew, especially for those produced in sub-optimal (low altitude) by promoting slower and balanced fillings and uniform ripening to berries, however; shade increased sugar concentration, which is an important factor or creating aroma of coffee deteriorating.

Apart from agronomic practice, cup quality is influenced by the ages of the plants. The samples from young trees are likely to be mild and thin, but fine in flavor while samples tree from old trees produces strong, taste and harsh characteristics brew. Medium aged trees bear with good flavor as well as acidity and body (Adugna, 2007). 
Anwar (2010) reported that the main factors affecting natural coffee quality is harvesting methods. It is widely agreed that traditional hand picking and husbandry labor as opposed to mechanical harvest, produces the best quality green coffee by decreasing the percentages of defects in coffee batches. On the other hand, Elias (2005) stated that low caffeine content is found in bean harvested at immature stage/unripe while over ripe coffee beans conventional analysis high performances liquid chromatography, this could be associated with slow metabolism of caffeine and its biodegradation at immature and over ripe stage of fruit development respectively.

\subsubsection{Post harvest factors}

Post-harvest processing of coffee is very important activity in coffee production and quality determinations (Willson, 1999). Coffee is either processed by wet and dry methods which vary in complexity and expected quality of coffee. Girma et al (2007) reported that both sun-drying as well as wet processing methods are operated in Ethiopia, which accounts for 70 percent and 30 percent of coffee produced in the country, respectively. Accordingly wet processed Arabica coffee is aromatic with fine acidity and some stringer, while dry processed Arabica coffee is less aromatic and less acidic but with greater body. The perceived acidity of washed coffee is significantly higher than the acidity found in naturally/dry processed coffee, this likely due to an increases in the body of naturally processed coffee is relative to wet processed coffee since some body masks coffee acidity (Clarke and Macrea,1989). The majority of coffee is prepared using dry processing system which the first method by which the fresh cherries are harvested and sun dried as whole.

The second method is the wet processing method in which the fresh red cherries are processed in three steps. i.e. removal of pulping mucilage, fermentation and washing and drying of parchment coffee (Clarke and Macrea., 1989 In washed coffee production, the final quality among others is greatly dependent up on the fermentation process/natural fermentation of coffee is the function of many parameters, such as environment, $\mathrm{PH}$, temperature, micro flora, and variety difference in the ripe cherries used for pulping, its geographical and culture origin and minor variation in the processing methods (Elias, 2005).

\subsubsection{Storage}

In storage, dry coffee quickly deteriorates in quality unless proper measures have to taken. It is known that parchment coffee deteriorate less rapidly than coffee, but deterioration in quality occur rapidly under unfavorable condition. Under storage, mostly quality deterioration occurs due to increases of moisture content of the bean, spoiling of the raw appearance of the bean by loss of colour fading or tainting or introduction of unpleasant flavors by infestation of storage insects (Behilu et al., 2007; Mekesha, 2007)

\subsubsection{Disease and insect pests}

Insect pests and diseases attack can affects the cherries directly or indirectly then cause coffee cherries deteriorate by debilitating the plants which will then produce immature or damage fruits. Insects like leaf miner and mites may also result in lower quality beans. For instance, as reported by Girma et al (2007) the coffee borer (hypothenemus hampii) feeds and reproduces inside coffee beans causes their quality to deteriorate. The Antestia bug serve as a vector for microorganism's transportation, in addition to the insect cause direct damage to the coffee cherries and bean finally resulted with a bitter flavor. Similarly, the fly Ceratistis capitata feeds on the mucilage and the cherries become infected with microorganisms, the secondary bacterial infections causes distinct potato flavor (Mekesha, 2007)

\subsubsection{Genetic factors}

The genetic origin of plant species greatly influences coffee quality. Genetic make up of coffee compared four traits (acidity, body, flavor and aroma) that over all stand for their suitability as selection criteria for genetic improvement of overall liquor quality (Bayeta, 2001). Furthermore Abiyot (2008) reported that, cup quality can be determined by different coffee genotypes with the assistance of professional coffee tasters. Similar finding also stated by Bezawit (2010) significance of genotype cross environmental interaction effects on coffee bean and finally on liquor quality of the product.

\subsection{Institutional Factors}

The national coffee board of Ethiopia was the first institution which responsible for coffee that established in 1957 with the aim of up grading of coffee quality, stimulating cooperative production, establishing market association, conducting research and dissemination of information on the coffee production, processing and marketing. Then after, the plantation in the south western parts of the country was organized under south western agricultural development organization (Elias, 2005). In 1993, the Ethiopian coffee market corporation was restructured in to two enterprises the Ethiopian coffee purchase and sale and the Ethiopian coffee export enterprises. In general, inappropriate and low institutional support in Ethiopia has greatest impact on coffee quality and production

\subsubsection{Socio- economic factors}

Factors that determine the adoption of technologies are more complex in case of perennial crops like coffee than in case of annual crops. This because of the difficulty in securing the benefits associated to the technologies due to the gaps and nature of the commodity trade which is influenced by international market (Girma, 2007). The age, gender, family size extension contact, attendances of training and experiences in coffee farming significantly 
affects the farmers' perception, which also directly affects the final quality coffee produced. According to Admasu et al. (2007) the sex of households and educational level has an impact on the final coffee produced and quality.

\subsubsection{Methods of coffee quality assessing?}

\subsubsection{Bean physical quality}

Internationally very low coffee prices that resulted from surplus production in the late 1990 and early 2005 have brought calls for quality to be increased in the market. International coffee organization has passed a resolution to this effect (Girma, 2007).In 2002 the international coffee organization is implementing the coffee quality improvement program with recommendation to export countries. According to the program it is not recommended to export with following characteristics for coffee Arabica, in excess of 86 defects per 300 grams sample and for Robusta in excess of 150 defects per 300 grams (Kebebe, 2009). On the other hand in Ethiopia the overall standard for raw and liquor quality grades of washed coffee ranges from 1 to 5 , where grade $1=81-100 \%$, grade $2=61$ $80 \%$, grade $3=41-60 \%$, grade $4=21-40 \%$ andgrade $5=20 \%$ or more than two defects .For unwashed coffee, the grades range from 1 to 5 , where grade $1=81-100 \%$, grade $2=63-80 \%$, grade $3=50-62 \%$, grade $4=31-49 \%$, or 2 cup defects, grade $5=15-30 \%$ or more than 2 cup defects(CLU 2007)

\subsubsection{Organoleptic Quality}

Cup quality is done on organoleptically by panels' of experienced coffee tasters and determined on the basis of the level of acidity, body and flavor. Cup quality or liquor quality an important attribute of coffee and act as stick for price determination (Wiley and Sohns, 2001). The organoleptic quality is affected by roasting i.e. according to the profile of temperature and length of roasting the tests and flavor perceived in the beverages will be different. The smell of the ground roasted coffee before water is added some times gives fragrance, then one can smell the aroma, evaluate the body and perceive the taste and flavors (Anwar, 2010). The aroma is responsible for all flavor attributes other than the mouth feel and sweet, salt, bitter and sour taste attribute that are perceived by tongue. Therefore the aroma of coffee is the most important attributes of specialty coffee (Behilu et al., 2008).

\subsubsection{Coffee Grading}

The primary issue of coffee grading is the country or region of origin, physical characteristics and standard taste. There is no universal coffee grading system except the recommended standard. Each coffee producing country has its own national standard of coffee grading scheme. In Ethiopia, there are two major components of coffee quality inspection. These are green analysts (visual test) and liquor analysis (cup test). These two methods are universally acceptable methods in both coffee producing and consuming countries tailored to the quality control system of respective country, from the total grading of coffee, the weight of green analysis is 40 percent and the remaining 60 percent is by cup test (Endale, 2007).

Green analysis: is based on human sense of sight (eye) and with the help of other techniques to identify and classify coffee. This method inspects the physical properties of coffee like shape, size, color, and uniformity/irregularity and defect count of coffee bean. In coffee grading, the parameters moisture content is $11.5 \%$ and screen size analysis is done by the help of sieve like apparatus to check the size of each coffee bean (Endale, 2007).

Cup test: it is based on human sense of test (tongue) to identify and classify coffee. It investigates the chemical properties of coffee. The parameters of cup test are acidity, body and flavor. Acidity is a primary coffee test sensation, created as acid in coffee combine with sugars to increases the overall sweetness of the coffee while the body is the texture and sensation of coffee in mouth. Flavor is an aroma or the smell perception of the elements presented in roasted coffee. Each of these parameters accounts $20 \%$ of total weight of coffee grading (Bezawit, 2011).

\subsubsection{Coffee Grading System}

According to the definition of Quality and Standard Authority of Ethiopia (QSAE), quality concept has different meaning, though complement each other, which are pointing in to some concepts. Quality is conformance with requirements or fitness for use in which the practice involved in the industry (customer, processor, and supplies etc) should agree on the requirements and of quality best suite our purpose. In line with notion stated earlier, coffee quality control and auction center was established with key objective of maintaining coffee quality control, which in turn facilitate the coffee marketing systems to be standard based and for betterment/proper functioning of long coffee supply chain of Ethiopia (Elias, 2007).

Sampling: is one of the main procedures in coffee quality assessment in which a sample drawer is expected to draw a representative/actual sample from each bags based on the sampling rule designed for the institution. In terms of the size, a sample drawer is subjected to draw $3 \mathrm{~kg} / 10$ tons of a truck which usually is the quantity that an average lorry/truck could carry at interval.

Coding: is under taking in which a person is responsible to assign an arbitrary code (an identify number, an alphabet, or combination of the two) which is secured and is only known the assigner. This is just the mechanism of accountability and transparency in which the experts do not know to whom that coffee belongs so that there will not be an opportunity for an expert to make a biased judgment (Endale, 2007). 


\subsubsection{Quality parameters for green coffee bean analysis}

Screen analysis: is important to make size assessment/grading. Based on outcome, one can easily know as to the sample size (large, bold, medium, small and etc) and it is conducted manually or electrically. Moisture content analysis: the other important aspect in the raw analysis is moisture testing which is very important in trying to know whether the coffee sample tested is with in permissible ranges/standard or not in which one sample is expected to have a maximum of $12 \%$.similary this can be carried out in laboratory assistant taking $300 \mathrm{gm}$ of green sample from whole sample.

Defect count system: this is very decisive and convectional accepted sub quality control parameter in coffee grading control. It is the principles of counting different kinds of coffee defects using a set of a standard developed, taking their rate of effect on overall quality of coffee. Internationally a standard is fixed for these green defects (over-ripe, foxy, under ripe immature, blacks, whites, stones, soil, earthy, broken and etc) but black and white taken as a bench mark. This activity is under taken $300 \mathrm{gm}$ of coffee from the whole (Endale, 2007).

Appearance/color: the overall performance (bluish, grayish, greenish, faded, whitish and etc) is analyzed by the expertise against the standard. For a better coffee (sample), the blue to grayish signifies the most desirable attribute of appearance/color.

Shape and make: the attribute is an interchangeable used term, which usually refers to structure or make up of the beans. The regions where coffee growing type and production systems persist are some of the factors that govern the shape and make quality of beans.

Roasting: coffee roasting is a chemical process by which aroma, acid, and other flavor components are either created/altered in way that should augment the flavor ,acidity after taste and body of the coffee as desired by the roaster or users. To attain such objective the degree of roasts is the desirable standard. Alemayehu et al (2008) mentioned that variation in moisture content, amount of coffee used and variation in the heating of cylinders and most important coffee types can make difference in duration and nature of roasted coffee.

Grinding: is physical change or an alteration in form with a means of reducing the size by crushing, rubbing, grafting, cutting, tearing and other process that will cause particle size reduction. The grinding required for cup tasting is termed medium that is proper for extraction/brewing of the liquor (CLU, 2007). On the other hand in Ethiopia the overall standard for raw and liquor quality grades of washed and unwashed coffee ranges from 1 to 9 where their grade are mentioned as the following table.

Table 1 Coffee graduation (both washed and sun-dried coffee)

\begin{tabular}{ll}
\hline Grade & Total Value (raw value + cup quality value) \\
\hline Grade 1 & $91-100$ \\
Grade 2 & $81-90$ \\
Grade 3 & $71-80$ \\
Grade 4 & $61-70$ \\
Grade 5 & $51-60$ \\
Grade 6 & $41-50$ \\
Grade 7 & $31-40$ \\
Grade 8 & $21-30$ \\
Grade 9 & $11-20$ \\
\hline
\end{tabular}

Source : (ICO, 2011)

Tabel 2. Standard parameter and their respective values used for washed coffee raw quality evaluation (CLU;2007) Raw value $(40 \%)$

\begin{tabular}{|l|l|l|l|l|l|}
\hline Shape \& make & points & color & point & odor & point \\
\hline Very good & 15 & Bluish & 15 & Clean & 10 \\
\hline good & 12 & Grayish & 12 & Trace & 8 \\
\hline Fair/average & 8 & Greenish & 8 & Light & 5 \\
\hline mixed & 5 & Faded & 5 & Moderate & 2 \\
\hline small & 2 & brownish & 2 & strong & 1 \\
\hline
\end{tabular}

Grade range: $1=81-100,2=61-80,3=41-60,4=21-40$ or $1-2$ defective cup,5-20 or more than 2 defective cup:foul,earthy, and chemical). 
3.Table standard parameter and their respective value used for washed coffee liquor quality evaluation (CLU,2007) Liquor value $(60 \%)$

\begin{tabular}{|l|l|l|l|l|l|}
\hline Acidity & Point & body & point & flavor & point \\
\hline Pointed & 20 & Full & 20 & Very good & 20 \\
\hline $\begin{array}{l}\text { Medium } \\
\text { pointed }\end{array}$ & 15 & Medium full & 15 & Good & 15 \\
\hline Medium & 10 & Medium & 10 & Average & 10 \\
\hline Light & 5 & Light & 5 & fair & 7 \\
\hline lacking & 2 & Thin & 2 & & \\
\hline
\end{tabular}

Grade Range: $1=81-100,2=61-80,3=41-60,4=21-40$ or $1-2$ defective cup, $5=20$ or more than 2 defective cup (defective cup:foul, earthy, and chemical).

\section{SUMMARY AND CONCLUSION}

Coffee Arabica is an economically important horticultural crop which is contributing the highest of all export revenue in Ethiopia. Coffee being the major crop of Ethiopia and it is produced almost all regions of the country serves as a major means of income for the live hood of coffee farming families. The coffee production in Ethiopia is the critical to Ethiopian economy with about quarter of total population depending directly or indirectly on their live hood. But, coffee production in Ethiopia is mainly concentrated in the south, southwest and east of the country. The annual production ranges from 200,000 to 250,000 metric tons depending on weather and soil condition. Coffee is produced by four methods in Ethiopia: forest coffee, semi-forest coffee, garden coffee and plantation coffee which they account $10 \%, 35 \%, 50 \%$ and $5 \%$, respectively. Despite favorable climatic conditions variety of local coffee types for quality improvement and long history of production in Ethiopia, coffee quality is declining from time to time due to several improper pre and post-harvest management practice. This still practiced by the majority of coffee producers/farmers, from which larger proportion of the product is obtained; these quality and organoleptic quality to give the final grade. The total grading of coffee, the weight of green analysis is 40 percent and the remaining 60 percent is by cup test.

\section{REFERENCE}

Abiyot Tessema. 2010. Characterization of some promising coffee (Coffee Arabica L) germ plasm collection for bean physical organoleptic and some biochemical quality attributed at Jimma.Msc Thesis, Submitted to Post graduate school of Jimma University College of Agriculture and Veterinary Medicine

Admasu Shibru, Zekarias Shumeta and Tsegaye Giday. 2007. Adoption of improved Coffee production technologies in Ethiopia. In proceeding of a national work shop four decades of a coffee research in August 14-17, Addis Ababa, Ethiopia pp 3007

Adugna Debele. 2007. Physiological effects of shade tree on growth and production of organic coffee.Msc Thesis, Wageningen University, and Netherland.

Alemayehu Teshoma, Esayas Kebede, Kassu Kebede.2008.Coffee development marketing improvement plan.

Anwar Abasanbi. 2010. Evaluation of Coffee quality and its problem in Oromia regional state. Msc Thesis, Jimma University College of Agriculture and Veterinary Medicine.

Bayetta Bellachew. 2001. Arabica Coffee breeding or yield and resistance to Coffee berry diseases. Msc Thesis, Jimma University College of Agriculture and Veterinary Medicine

Behailu Weldesenbet, Abara Shaleh, Nugussie Mokennen and Solomon Indris. 2007. Coffee processing and quality research in Ethiopia. In proceeding of a national work shop four decades of coffee research in August 14-17, Addis Ababa, Ethiopia pp 307-316.

Bezawit Teklu. 2011. Effects of processing methods and drying materials on bean physical and sensory quality attributes of coffee (Coffee Arabica L).Msc Thesis, Jimma University College of Agriculture and Veterinary Medicine.

Clarke R.J. and Macrae. R. 1989. Coffee Technology .University of Reading, UK.

CLU.2007.Training for Trances Coffee Quality Inspection and Auction Center, Addis Ababa, Ethiopia.

Elias Abebe. 2005. Eonomics of Coffee Bean marketing in Goma District in Jimma Zone of Ethiopia.MSc, Thesis, submitted to school of graduate studies, Alemaya University, Ethiopia.

Endale Asfaw. 2007. Physical quality standard and grading systems of Ethiopian coffee in demand supply chain. In proceeding of a national work shop four decades of coffee research in August 14-17, Addis Ababa, Ethiopia pp 328.

FAO.2008.Special report WEP Crop and food supply assessment mission to Ethiopia, January 24-25 Addis Ababa, Ethioipia.

Fayera Senbeta. 2006. Biodiversity and Ecology of a formontane rain forest with Wild Coffee Arabica L in Ethiopia.PHD, Dissertation University of Bonn, and REBonn

Girma Adugna, Demelash Teferi, Chala Tefuka, Sisay Tesfaye and Arega Zeru. 2007. Research finding in Coffee 
Wilt diseases. In proceeding of a national work shop four decades of coffee research in August 14-17, Addis Ababa, Ethiopia pp250-260

Girma Tamiru. 2007. Technology transfer and adoption by coffee growers. In proceeding of a national work shop four decades of coffee research in August 14-17, Addis Ababa, Ethiopia pp 411.

ICO.2011.Coffee quality Improvement Program Implementation, Resolution February, 2011 Addis Ababa, Ethiopia.

John Wiley and Sohns.2001.Coffee Flavor Chemistry .Newyork, USA.

Kebebe Assefa and Woldeyesus Sinebo.2009.In Proceedings of the $13^{\text {th }}$ Annual Conference of the Crop Science Society of Ethiopia, Addis Ababa, Ethiopia.

K.C.Willson.1999.Coffee, Cocoa and Tea. University of Liver Pool, UK.

, D.J.1997.The Plant Book. Mabberely Cambriddge University, Cambridge

Mekesha Chichaybelu. 2007. Seasonal Abundance of Artesian bugs (Antestiopsis intricate) in south west of Ethiopia. In proceeding of a national work shop four decades of coffee research in August 14-17, Addis Ababa, Ethiopia pp291-295

Taye and Tesfaye. 2002. Organic Coffee production hope for small scale farmers in Ethiopia. Proceeding of the $19^{\text {th }}$ International conference on Coffee Science, Trieste Italy.

Tena Gobena .2008.Coffee Production Systems as tool for rehabilitation of degraded land in case of Haru.Msc Thesis, Jimma University College of Agriculture and Veterinary Medicine.

Workafes Woldestadik and kassu Kebede 2000. Coffee production systems in Ethiopia. Inof Proceedings of the workshop on control of Coffee Berry Disease (CBD), August 13-15, 1999, PP. 99-106, Addis Ababa, Ethiopia. 